\section{Is Screening for Suicidal Risk and Hazardous Drinking Possible in a Level 1 Trauma Center?}

\section{Brian G Celso ${ }^{1 *}$, David Chesire ${ }^{2}$, Andrew Kerwin ${ }^{2}$ and Joseph J Tepas ${ }^{2}$}

'Department of Psychiatry, University of Florida, Jacksonville, FL, USA

${ }^{2}$ Department of Surgery, University of Florida, Jacksonville, FL, USA

\begin{abstract}
The aim of this study was to investigate if screening for suicide risk and hazardous drinking was possible at a Level 1 trauma center. 107 trauma patients were screened using the Risk for Suicide Questionnaire (RSQ) and Alcohol Use Disorder Identification Test (AUDIT). Three questions from the AUDIT were compared to the full 10 question AUDIT to assess the use of a rapid screening tool to detect alcohol misuse among trauma patients. Results showed that the RSQ identified one case of deliberate self-injury and 3 cases with recurrent thoughts of suicide. Correlation between the AUDIT and AUDIT-3 was 0.904, $(p<0.01)$. On the AUDIT, the cutoff score that identified hazardous drinking based on criteria of a positive risk for suicide was 1 with a sensitivity of $75.0 \%$ and specificity of $64.2 \%$. Likewise, the AUDIT-3 cutoff scores that identified hazardous drinking was 1 with the same sensitivity of $75.0 \%$ but a higher specificity of $75.5 \%$. A Receiver Operator Characteristic curve of the AUDIT-3 compared to the full AUDIT showed the areas under the curve of 0.56 and 0.55 , respectively $(p=0.97)$. This study demonstrated that rapid screening for suicide risk and hazardous drinking was possible at a Level 1 trauma center.

Keywords: AUDIT; Alcohol screening; RSQ; Suicide; Trauma
\end{abstract}

\section{Introduction}

Persons who attempt suicide are frequently transported to Emergency Departments (ED), often hospitalized, and many subsequently transferred to a psychiatric facility [1]. The most serious suicide attempts by such violent means as knife or gun-shot are likely to be treated at a state designated Level 1 trauma center and staffed by those specially trained to handle the most severely injured. As

${ }^{*}$ Corresponding author: Brian $\mathrm{G}$ Celso, $\mathrm{PhD}$, Department of Psychiatry, University of Florida, Jacksonville 580 W. $8^{\text {th }}$ St., Tower II, $6^{\text {th }} \mathrm{Fl}$., Suite 2005, Jacksonville, FL 32209, USA, Tel: 904-244-3996; E-mail: Brian.Celso@jax.ufl.edu

Citation: Celso BG, Chesire D, Kerwin A, Tepas JJ (2014) Is Screening for Suicidal Risk and Hazardous Drinking Possible in a Level 1 Trauma Center? J Emerg Med Trauma Surg Care 1: 003.

Received: October 30, 2014; Accepted: December 07, 2014; Published: December 22, 2014 trauma center staffs are often the first to encounter suicidal patients, this raised the question if screening for suicide risk and hazardous drinking was possible at a busy Level 1 trauma center with a minimum of time, effort and resources.

\section{Objectives}

Self-injury in the state of Florida has continued to rise for the last decade. In Duval County, admission for a self-inflicted injury in 2010 exceeded the state trend for the same year (Figure 1). A state designated Level 1 trauma center in Duval County, Florida served as the site to recruit trauma patients for participation in the study. The busy trauma service treats over 4,000 injured patients each year from counties in Northeast Florida and Southeast Georgia. The three objectives of the present research were first to investigate if it was possible to administer a rapid screening instruments to assess for risk of suicide and hazardous drinking within a high risk trauma population. The second objective was to compare the screening methods used to identify those trauma patients at risk for suicide behavior and hazardous drinking with past efforts for targeted interventions. The third objective was to contact all study participants in one month from the time of their initial screening to determine the follow-up rate after hospital discharge.

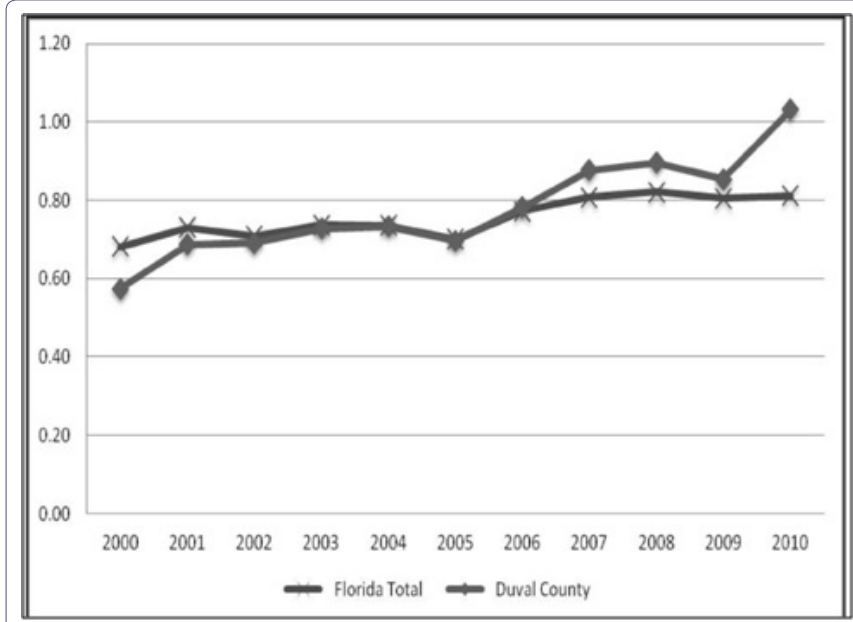

Figure 1: Self-harm rate for Florida and Duval County per 1000 pop (20002010).

\section{Materials and Methods}

\section{Participants}

Participants were patients ages 18-years-old and older registered under the Trauma Service at a Level 1 trauma center in Duval County, Florida. The name, age, gender, ethnicity and marital status were collected on all study participants. Two phone numbers were requested to contact the participant in one month. Inclusion criteria were that the patient was admitted to a medical floor from the trauma resuscitation area. Patients were excluded from participation if they were admitted with a Glasgow Coma Scale of 12 or less, required intensive care for longer than 48 hours and/or intubation for longer than 48 hours. 


\section{Measures}

Risk of suicide: Trauma patients were assessed for suicide risk with a modified Risk of_Suicide Questionnaire (RSQ). The 4 questions on the RSQ are; Are you here because you tried to hurt yourself?, In the past week, have you been having thoughts about killing yourself?, Have you ever tried to hurt yourself in the past (other than this time)?, Has something very stressful happened to you in the past few weeks (a situation that was very hard to handle)? An additional item was included for this study to inquire about family members' past suicide behavior. The questions are answered with either a "yes" or "no" response. A "yes" answer or having "no response" accompanied by nonverbal behaviors of concern to any of the RSQ four questions constitutes a positive screen [2].

Hazardous drinking: The Alcohol Use Disorders Identification Test (AUDIT) was administered to trauma patients to screen for hazardous drinking [3]. The AUDIT consists of 10 items scored from 0 to 4 for a maximum score of 40 with higher scores reflecting more alcohol misuse. A score of 8 is the recognized cutoff for hazardous drinkers computed for positive criteria that include, average daily alcohol consumption, recurrent intoxication, presence of at least one dependence symptom, diagnosis of alcohol abuse or dependence, and self-perception of a drinking problem. Three questions from the AUDIT, the AUDIT-3, were analyzed separately to assess their effectiveness as a screening tool for alcohol misuse in the trauma population: How often do you have a drink containing alcohol? How many drinks containing alcohol do you have on a typical day when you are drinking? How often do you have six or more drinks on one occasion? Gordon et al. [4] found the AUDIT-3 was strongly correlated with alcohol consumption per week. Sensitivity and specificity for hazardous drinking in relation to the diagnosis of risky drinking were $92.4 \%$ and $74.3 \%$ for men and $90.9 \%$ and $68.4 \%$ for women with cut off scores of 5 and 4, respectively [5].

\section{Procedure}

After Institutional Review Board approval, new admissions to the trauma service were approached by a member of the trauma team within the first 48 hours of admission. A prepared statement was read to the patients to recruit them for participant in this research. The patients that agreed to participate were later visited by the Principal Investigator (PI) to obtain informed consent and to answer any questions about the study. The PI maintained the original signed consent form and a copy of the form was given to all participants. Both the RSQ and AUDIT were administered at the patient's bedside and their responses recorded. A progress note indicating that the patient was recruited for the study was placed in the medical record. The Principal Investigator also collected two phone numbers provided by the participant to contact the patient one month from the time of their screening.

Those participants who screened positive as at-risk for suicide by an affirmative response to either Question 1 or 2 on the RSQ triggered a consultation request to the Psychologist in the Department of Surgery to perform a comprehensive evaluation and assess for current suicidality. The Trauma Psychologist determined the appropriate referral for the patient and ensured that the disposition included instructions for follow up. Any study participant determined to be at immediate risk for self-harm at the time of discharge was transferred to a psychiatric facility if no other less restrictive solution to a safe discharge was available. Those not considered at imminent risk of intentional self-harm were referred to either their primary care physician or to a community mental health center for follow up as appropriate. Patients at risk for hazardous and harmful alcohol use were referred to an area substance abuse treatment facility and Alcoholics Anonymous to address their alcohol misuse. At one month from the time of screening, each participant was telephoned with the numbers provided to determine how many were available for follow up.

\section{Statistical analyses}

The demographic data were analyzed for patient characteristics that described the population of interest. An Eta correlation was conducted to determine the association between alcohol misuse and suicidal behaviors among the trauma population. The AUDIT-3 was analyzed along with the AUDIT to show the usefulness of a 3 question rapid screening tool to detect alcohol misuse among trauma patients when compared to the full 10 questions from the AUDIT as the standard. Receiver Operator Characteristic (ROC) curve analyses were performed to test the statistical significance of the difference between the areas under the two ROC curves and the sensitivities for different specificities of the AUDIT- 3 based on criteria of a positive risk for suicide to identify hazardous drinking among male and female trauma patients. The follow up rate for study participants was calculated as a percentage of the number of patients successful contacted after one month from the total of trauma patients screened. Alpha level was set at 0.05 . Analyses were calculated using SPSS Version 19 (IBM SPSS, Armonk, NY) and Medcalc Version 12 (MedCalc Software, Mariakerke, Belgium).

\section{Results}

\section{Demographics}

Study participants included 107 trauma patients admitted between June and October 2011. The average age of the participants was 44.3 years with a standard deviation of 18.6. African Americans comprised $27 \%$ of the sample, Asians 1\%, Caucasians $67 \%$ and Hispanics $3 \%$. Two percent of the participants listed their ethnicity as other. Seventy two percent of the participants were men and $28 \%$ women. Forty four percent were single, $32 \%$ were married, $12 \%$ were divorced, $6 \%$ were separated and 7\% were widowed. A total of 1525 adult patients were admitted under the trauma service during the five month study period. Thus, the sample represents 7 percent of those who were treated.

\section{Risk of suicide}

On the RSQ, of the 107 participants, the modal affirmative response was a stressful event occurring in the past few weeks. One participant reported a deliberate self-injury and three endorsed thoughts of suicide in the past week. The method of self-injury was by knife wound to the forearm. The four patients who screened positive for risk of suicidal behavior were referred for trauma psychology follow up. Seven participants reported an immediate family member attempted or completed suicide. However, none of them were among the positively screened patients. An Eta correlation performed between the RSQ and AUDIT- 3 produced a correlation of 0.323 . Seventy percent of the study participants were able to be contacted one month after their initial screening. Two of the four patients who screened positive for suicide risk were able to be contacted at follow-up. 


\section{Hazardous drinking}

The mean score for the AUDIT was 5.8 with a standard deviation of 7.5. The mean score for the AUDIT- 3 was 3.4 with a standard deviation of 3.4. Correlation between the 10 question AUDIT and AUDIT-3 was 0.904, $(\mathrm{p}<0.01)$. The ROC curve of the AUDIT-3 compared to the ROC curve from the 10 question AUDIT showed areas under the curve of 0.562 and 0.551 , respectively. The difference between the areas was not significantly different $(p=0.9672)$. The ROC curves are presented in Figure 2. For this sample, on the AUDIT, the cutoff score that identify hazardous drinking among male and female trauma patients based on criteria of a positive risk for suicide was 1 with a sensitivity of $75.0 \%$ and specificity of $64.2 \%$. For the AUDIT-3, the cutoff scores that identify hazardous drinking based on a positive risk for suicide was also 1 with the same sensitivity of $75.0 \%$ but a higher specificity of $75.5 \%$. Of note, one patient who scored above the cutoff was placed on DT prophylaxis to prevent withdrawal symptoms during hospitalization.

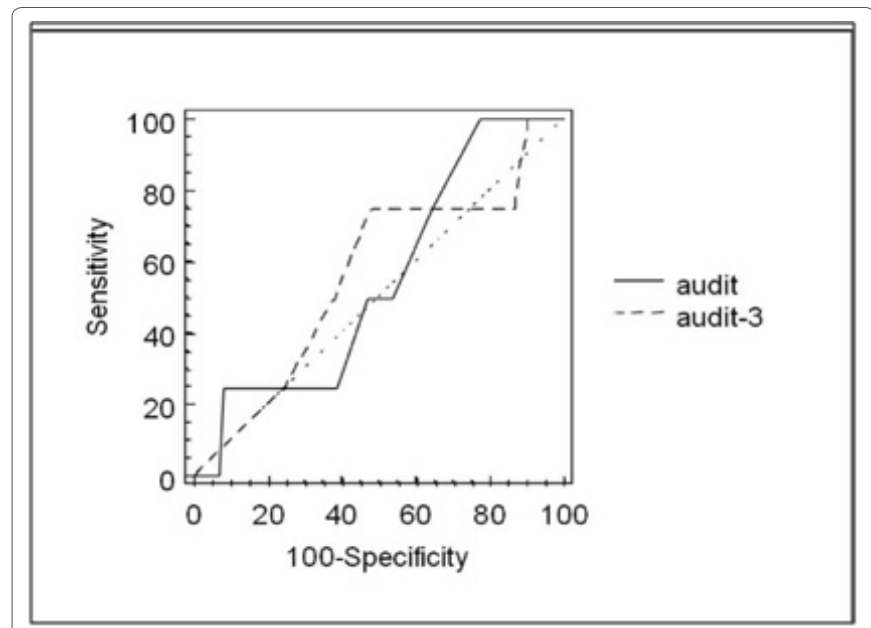

Figure 2: Receiver operator characteristic curves for AUDIT vs. AUDIT-3.

\section{Discussion}

The main purpose of the present study was to investigate the screening for suicide risk and hazardous drinking at a Level 1 trauma center. The majority of participants reported a recent stressful event given that they were involved in a trauma and currently hospitalized. The AUDIT scores in this trauma population were lower than the average score for hazardous drinking. Rapid screening of trauma patients with shorter versions of valid and reliable measures reduced the amount of time, effort and resources required. In addition, rapid screening could be accomplished without creating discomfort to patients regardless of the degree of injury. Equally important was the finding that although the majority of trauma patients were contacted after discharge, $30 \%$ were lost to follow up if any intervention should have been required in the future. This study demonstrated that screening for risk of suicide and hazardous drinking is possible at a busy Level 1 trauma center.

Folse et al., [6] had a $30 \%$ positive screen rate, while the present study only had a $3 \%$ positive screen rate. One explanation may be that while Folse et al., [6] conducted their study in an ED, this study screened trauma patients who likely represent an entirely different population. Hospital EDs admit patients ranging from self-reporting suicidal thoughts through acting on the thoughts by ingesting substances, asphyxiation and cutting and thus have a higher prevalence. Trauma patients admitted for a suicide attempt, on the other hand, select lethal means of suicide but fail to complete the suicide and represent a much lower percentage of trauma patients. The possibility also exists that some of the trauma patients who were screened underreported their suicidality, hazardous drinking or both. This may account for the lower sensitivities and specificities calculated based on the overall risk for suicide and thus the majority of participants in the study scored below the established cutoffs for hazardous and harmful drinking for the AUDIT and AUDIT-3.

\section{Trauma and suicide}

Trauma patients exhibit pre-injury and post-injury factors that are similar to the risk factors associated with suicide behavior [7]. The average trauma patient tends to be a younger, white male with pre-morbid risk factors of disordered personality and substance misuse. Furthermore, the pre-injury personality traits such as impulsivity and aggression, the psychiatric condition of depression, and a positive alcohol test at admission were positively associated with suicide behavior. Post-injury factors of brain and spinal cord injury and posttraumatic stress also increased suicide risk for trauma patients.

The burden, that intentional self-harm poses to a trauma system is clearly illustrated in the western United States. Because self-inflicted injuries treated at western trauma centers most often involved violent mechanisms such as gunshot and stab wounds, the patients required a greater need for surgery, higher levels of medical support, and had a greater risk for complications [8]. In addition, self-inflicted injuries treated at western trauma centers had the highest mortality, longer days in the hospital, and most costly care when compared to assaults or unintentional injuries. This is a dilemma also faced by trauma systems across the country.

There has been a suspicion that single vehicle, single occupant road deaths may sometimes be disguised suicides $[9,10]$. This, however, was not borne out when comparisons were made for age, seasonal variation, and adverse road conditions. When followed over time, 55\% of those treated for unintentional injury were later hospitalized for a suicide attempt [11]. In addition, there was a three times greater risk for suicide among those involved in a single vehicle crash. Therefore, these high-risk injuries may have in fact been misclassified suicide attempts. Thus, motor vehicle crashes that involve serious damage and injury are an opportunity to screen for suicide risk in a trauma center.

Conwell et al. [12] listed the risk factors of older age, mood disorders such as major depression, social isolation, declining physical health, and disability that place adults at risk for suicide. Alcohol and substance use disorders, however, were not found to be prevalent among older suicide attempters. Older trauma patients, especially elderly white males, were more likely to use violent methods to attempt suicide such as firearms, and the attempts were more likely to be lethal [13]. Similarly, Crandall et al. [14] found that 75\% of older trauma patients admitted for suicide attempt used a firearm. However, only $2 \%$ had a known psychiatric diagnosis. The suicide attempt may represent the first signal of depression or suicidality among older adults. For these reasons, more attention to suicide risk screening with instruments such as the Folse, Eich, Hall and Ruppman [6]. Risk for Suicide Questionnaire (RSQ) that has been found to be an effective screen for both pediatric and adult populations should be considered. 


\section{Trauma and alcohol use}

Alcoholism was found to be the number one co-occurring disorder among trauma patients $[15,16]$. The use of screening tools such as the AUDIT, CAGE and the Short-Michigan Alcoholism Screening Test (SMAST) has been evaluated to determine their sensitivity and specificity to distinguish non-hazardous drinking from hazardous and harmful drinking. The AUDIT was shown to be most appropriate to screen for hazardous and harmful drinking whereas the CAGE and SMAST were better used for abusive and dependent drinkers in the younger adult patients [17] and older adults [18]. Soderstom et al. [16] investigated alcohol screening at a Level 1 trauma center and found the CAGE was able to detect alcohol dependence when compared to the standard of a structured diagnostic interview.

Ehrlich et al. [19] suggests that because there is a high prevalence of hazardous drinking among trauma patients, trauma centers are an ideal setting to screen for alcohol misuse. In the same way that screening and intervention for alcohol may likely provide secondary prevention of re-injury, a secondary survey for suicide risk may provide the same reduction in the incidence of suicide behavior. Suicide among trauma patients was shown to be higher than in the general population. In the state of Maryland, for example, Ryb et al. [7] showed that their trauma population was at an $89 \%$ higher risk for suicide than in the general population. Therefore, a trauma system lends itself as a vital link to reduce suicide risk by screening for the known risk factors for suicide and providing appropriate follow up to lower the likelihood of suicide after discharge.

Recognizing the time constraints of staff in busy EDs does not negate the necessity of screening. Trauma surgeons surveyed supported alcohol screening and brief interventions as part of routine care [20]. Screening initiatives that lead to appropriate treatment may not only help reduce the reoccurrences of intentional self-harm and hazardous drinking but also lead to decreased costs in the ED and improved staff morale. Medical staff training on assessment was shown to improve effectiveness of ED patients screening [21,22]. Reduction in the amount of time required to complete a risk assessment can be accomplished by the use of a screening check-list or a patient self-report form. Appropriate follow-up for intentional self-harm and substance use may include the referral to a general practitioner or mental health specialist to reduce the risk of reoccurrence [23].

Several limitations exist within this study. First, the participants were self-selected from a single urban center. Thus, the findings may not be representative of the true population of trauma patients most at risk for suicide or alcohol problems as these rates were shown to vary by state and region [8]. Second, the trauma patients who consented to be assessed were likely those who were at least risk. A frequent comment heard was that neither suicide nor alcohol was a problem for the person. Third, patients with a GCS less than 12 were excluded from the study and may represent a higher risk population. Finally, the responses of the participants were not verified by a corroborating person. Therefore, the low rate of positive screens was likely to affect the sensitivities of the two instruments and limit the generalizability of the findings.

Despite these limitations, since the use of screening instruments for detecting suicide risk and alcohol abuse was demonstrated to be possible within a Level 1 trauma center. Future research may include a large, hypothesis-driven study to construct a prediction model based on premorbid patient characteristics and injury data in order to better identify patients most at risk for self-harm and target appropriate interventions. Such a study should include multiple trauma centers and patients with a GCS less than 12 for improved generalizability. An unscreened control group would be used for comparison. All study participants contacted after discharge can be reassessed for present suicidal risk. Trauma patients screened positive as at-risk for suicide at the initial screening and referred for follow up can report if follow up occurred. A cross-validation may be conducted to verify the sensitivities and specificities of the screening instruments. The disease of trauma as well as mental illness afflicts men and women and members of all ages and ethnic racial groups alike. Designated Pediatric Trauma Centers designed to meet the special needs of younger patients may add an age-appropriate suicide screening to their assessment as well.

\section{Conclusion}

Trauma centers can play an important role in reducing the burden intentional self-harm places on the healthcare system. Our study indicates that screening of patients for suicidal risk and problem drinking in a Level 1 Trauma Center is feasible and effective and should be considered in all Trauma centers. It is hoped that the early recognition of suicidal behavior and hazardous drinking will lead to earlier referral and treatment, improved outcomes and prevention of further trauma. Trauma centers and associated systems all have a stake to increase awareness and prevention of suicide behavior.

\section{References}

1. Bittner JG $4^{\text {th }}$, Hawkins ML, Atteberry LR, Ferdinand CH, Medeiros RS (2010) Impact of traumatic suicide methods on a level I trauma center. Am Surg 76: 176-181.

2. Horowitz LM, Wang PS, Koocher GP, Burr BH, Smith MF, et al. (2001) Detecting suicide risk in a pediatric emergency department: development of a brief screening tool. Pediatrics 107: 1133-1137.

3. Saunders JB, Aasland OG, Babor TF, de la Fuente JR, Grant M (1993) Development of the Alcohol Use Disorders Identification Test (AUDIT): WHO Collaborative Project on Early Detection of Persons with Harmful Alcohol Consumption--II. Addiction 88: 791-804.

4. Gordon AJ, Maisto SA, McNeil M, Kraemer KL, Conigliaro RL, et al. (2001) Three questions can detect hazardous drinkers. J Fam Pract 50: 313-320.

5. Gual A, Segura L, Contel M, Heather N, Colom J (2002) Audit-3 and audit-4 effectiveness of two short forms of the alcohol use disorders identification test. Alcohol Alcohol 37: 591-596.

6. Folse VN, Eich KN, Hall AM, Ruppman JB (2006) Detecting suicide risk in adolescents and adults in an emergency department: a pilot study. J Psychosoc Nurs Ment Health Serv 44: 22-29.

7. Ryb GE, Soderstrom CA, Kufera JA, Dischinger P (2006) Longitudinal study of suicide after traumatic injury. J Trauma 61: 799-804.

8. Steljes TP, Fullerton-Gleason L, Kuhls D, Shires GT, Fildes J (2005) Epidemiology of suicide and the impact on Western trauma centers. J Trauma 58: 772-777.

9. Jenkins J, Sainsbury $P$ (1980) Single-car road deaths--disguised suicides? Br Med J 281: 1041.

10. Rockett IR, Spirito A, Fritz GK, Riggs S, Bond A (1991) Adolescent risk-takers: a trauma center study of suicide attempters and drivers. Int J Soc Psychiatry $37: 285-292$.

11. Grossman DC, Soderberg R, Rivara FP (1993) Prior injury and motor vehicle crash as risk factors for youth suicide. Epidemiology 4: 115-119. 
12. Conwell Y, Lyness JM, Duberstein P, Cox C, Seidlitz L, et al. (2000) Completed suicide among older patients in primary care practices: a controlled study. J Am Geriatr Soc 48: 23-29.

13. Hadjizacharia P, Brown CV, Teixeira PG, Chan LS, Yang K, et al. (2010) Traumatic suicide attempts at a level I trauma center. J Emerg Med 39: 411418

14. Crandall M, Luchette F, Esposito TJ, West M, Shapiro M, et al. (2007) Attempted suicide and the elderly trauma patient: risk factors and outcomes. J Trauma 62: 1021-1027.

15. Morris JA Jr, MacKenzie EJ, Edelstein SL (1990) The effect of preexisting conditions on mortality in trauma patients. JAMA 263: 1942-1946.

16. Soderstrom CA, Smith GS, Kufera JA, Dischinger PC, Hebel JR et al. (1997) The accuracy of the CAGE, the Brief Michigan Alcoholism Screening Test, and the Alcohol Use Disorders Identification Test in screening trauma center patients for alcoholism. J Trauma 43: 962-969.

17. McCusker MT, Basquille J, Khwaja M, Murray-Lyon IM, Catalan J (2002) Hazardous and harmful drinking: a comparison of the AUDIT and CAGE screening questionnaires. QJM 95: 591-595
18. Fink A, Tsai MC, Hays RD, Moore AA, Morton SC et al. (2002) Comparing the Alcohol-related Problems Survey (ARPS) to traditional alcohol screening measures in elderly outpatients. Arch Gerontol Geriatr 34: 55-78.

19. Ehrlich PF, Maio R, Drongowski R, Wagaman M, Cunningham R et al. (2010) Alcohol interventions for trauma patients are not just for adults: Justification for brief interventions for the injured adolescent at a pediatric trauma center. J Trauma 69: 202-210.

20. Schermer CR (2005) Feasibility of alcohol screening and brief intervention. J Trauma 59: S119-123.

21. Crawford MJ, Turnbull G, Wessely S (1998) Deliberate self harm assessment by accident and emergency staff--an intervention study. J Accid Emerg Med 15: 18-22.

22. Dennis M, Evans A, Wakefield P, Chakrabarti S (2001) The psychosocial assessment of deliberate self harm: using clinical audit to improve the quality of the service. Emerg Med J 18: 448-450.

23. Mitchell AJ, Dennis M (2006) Self harm and attempted suicide in adults: 10 practical questions and answers for emergency department staff. Emerg Med J 23: 251-255. 